\title{
The effect of a breakfast containing multiple functional ingredients on glucose response, insulin response and cognitive performance in adolescents
}

\author{
S. Kennedy ${ }^{1}$, L. Ryan ${ }^{2}$ and M.E. Clegg ${ }^{1}$ \\ ${ }^{1}$ Functional Food Centre, Department of Sport and Health Sciences, Oxford Brookes University, Gipsy Lane, Oxford, \\ OX3 OBP and ${ }^{2}$ School of Science and Computing, Galway-Mayo Institute of Technology, Dublin Road, Galway, \\ Ireland
}

Adolescent breakfast studies generally support an association between eating a regular breakfast and a reduced risk of obesity, chronic disease $^{(1)}$ as well as benefits to academic performance ${ }^{(2)}$ and cognitive function ${ }^{(3)}$. Fewer studies have considered breakfast composition and the relative effects on cognitive function and metabolic markers of health. Carbohydrate containing foods varying in quality or quantity can affect blood glucose levels differently which have been found to correlate with performance on cognitive tasks ${ }^{(4)}$. Blueberry consumption and other polyphenol rich foods have been linked with improvements in cognitive performance ${ }^{(5)}$ and have shown potential for improving glucose and insulin regulation ${ }^{(6)}$. The aim of the current study was to assess the impact of functional food ingredients, consumed at breakfast, to enhance glucose and insulin response and cognitive performance in adolescents.

Twenty-two children (13 girls, 9 boys) were recruited from two Oxfordshire Schools. Following an overnight fast upon arrival at school baseline blood glucose and insulin samples from finger-pricks were taken alongside spatial and verbal memory performance measures. Following the consumption of either a control breakfast or the functional food (FF) breakfast, further blood measures were taken at 15, 30, 45, 60, 90, 120, 150 and 180 minutes. Further cognitive measures (spatial working memory and a delayed recall task) were assessed at 60,120 and 180 minutes. Control and FF breakfasts were matched to contain $50 \mathrm{~g}$ available carbohydrate and similar amounts of energy, protein and fat. The FF breakfast contained blueberries, baobab extract, cinnamon and oats.

There were no significant differences between breakfast glucose area under the curve (AUCs) at T60, T120, or T180. However, there was a higher glucose peak after consumption of the control breakfast $(6.8 \pm 0.5 \mathrm{mmol} / \mathrm{L})$ compared to the FF breakfast $(6 \cdot 3 \pm 0.7$ $\mathrm{mmol} / \mathrm{L})(p=0.009)$. There were significant differences between insulin AUC values at T60 (control $2426.3 \pm 1098.6 \mathrm{vs}$. FF 1981.1 $\pm 1156.7 \mu \mathrm{U} / \mathrm{mL} / \mathrm{m})(p=0.046)$ and T120 (control $3792.3 \pm 1751.3$ vs. FF breakfast $3070.8 \pm 1879.1 \mu \mathrm{U} / \mathrm{mL} / \mathrm{m})(p=0.041)$ but not at T180. Furthermore, after consumption of the control breakfast there was a significantly higher insulin peak $(90.90 \pm 40 \cdot 26 \mu \mathrm{U} /$ $\mathrm{mL})$ compared to the FF breakfast $(72.68 \pm 37.57 \mu \mathrm{U} / \mathrm{mL}),(p=0.013)$. There were no effects of breakfast on cognitive performance for either the spatial map task or the delayed word recall task. There was a trend during the map task for the number of correctly answered items to decrease over the testing period and subsequently the number of wrong items to increase, however, this was not significant $(p=0.067, p=0.096$ respectively).

The addition of multiple functional food ingredients to a breakfast improved peak glucose, peak insulin and insulin response compared to a control breakfast in adolescents, however, there were no effects of different breakfasts on spatial or verbal memory, satiety or mood.

1. Wennberg M, Gustafsson PE, Wennberg P et al. (2015) Public Health Nutr 18, 122-9.

2. Littlecott HJ, Moore GF, Moore L et al. (2015) Public Health Nutrition 13, 1.

3. Hoyland A, Lawton CL'\& Dye L (2008) Neuroscience and Biobehavioral Reviews 32, 72-85.

4. Benton D \& Sargent J (1992) Biological Psychology 33, 207-210.

5. Krikorian R, Shidler MD, Nash TA et al. (2010) J Agric Food Chem 58, 3996-4000.

6. Coe SA, Clegg M, Armengol M et al. (2013) Nutr Res 33, 888-96. 